\title{
Laparoscopic repair in a case with Morgagni hernia
}

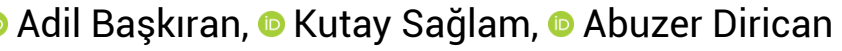 \\ Inönü University Institute of Liver Transplantation, Turgut Ozal Medical Center, Malatya, Turkey
}

\begin{abstract}
Morgagni hernia is herniation of the abdominal contents through a congenital retrosternal defect to the thoracic cavity. In the present study we report a case of 40 years old male patient who was admitted to our department with signs of intestinal obstruction and dyspnea and was diagnosed as incarcerated Morgagni hernia. The diagnosis was established with direct chest radiograms and thorax computerized tomography. Following the reduction of the omentum and the colon segment in the hernia, the defect was sutured primarily and the repair was reinforced by a dual mesh. In patients with constipation and obstipation possibility of presence of Morgagni hernia should be kept in mind and proper investigative measures should be obtained. Keywords: Congenital defect; intestinal obstruction; Morgagni hernia.
\end{abstract}

\section{Introduction}

Diaphragm is an anatomic barrier between thorax and abdominal cavity. It is formed between the 1st and 4th month of gestation together with truncal musculature, septum transversum, pleuroperitoneal membranes and dorsal mediastinal mesentery of the esophagus. ${ }^{[1]}$ Congenital diaphragmatic hernias are classified in to 3 subtypes according to the localization of the defect; Morgagni (posterolateral), Bochdalek (posterolateral) and septum transversum (central). Globally the prevalence of diaphragmatic hernias is one in 2000-12500 live births. ${ }^{[2]}$ Morgagni hernia is usually asymptomatic at birth and will not be diagnosed until later in adult life. Usual contents of the Morgagni hernia in descending order of incidence is omentum, colon, stomach, and small bowel. Incarceration is very rare in Morgagni hernia. Nevertheless researchers have proposed repair of the defect before any complications develop. ${ }^{[3]}$ Computerized tomography is the gold standard diagnostic modality and specificity has been reported to be ranging between $83-100 \%$. Surgical approaches involve mesh reinforcement or primary repair through laparoscopic, open or thoracoscopic approach. Since it's a rare clinical entity large volumes comparing the efficacy of different modalities are lacking. There are only small volume case series reporting favorable short-term outcomes. ${ }^{[3]}$ In the present study, we present case of 40 years old male patient who admitted to our department with dyspnea and recurrent intestinal obstruction and was diagnosed to have Morgagni hernia. Our diagnostic and therapeutic approaches are discussed in the guidance of the current literature.

\section{Case Report}

Forty-years old male patient was admitted to our department with nausea, vomiting and abdominal distention. The past medical history showed that he had no previous abdominal surgery. He expressed a progressively worsening in the last three years. He had been evaluated by 
some gastroenterologist 6 months prior to admission to the emergency department and a colonoscopy was prematurely terminated due to ineffective bowel preparation.

Physical examination revealed that the abdomen was tender and respiratory sounds in the right basal area were reduced. The laboratory workup was normal. Plain abdominal radiogram showed air fluid levels in the abdomen. Plain chest roentgenogram showed a mass in the right thoracic cavity showing air fluid level (Fig. 1a). Thorax computerized tomography showed a hernia sac extending from the para-cardiac area to carina region. The contents of the hernia were omentum and a segment of the colon (Fig. 1b, c).

The patient was diagnosed to have an irreducible diaphragmatic hernia and an operative intervention was planned. The operation started with the laparoscopic approach and 3 trocars were inserted: one $10 \mathrm{~mm}$ camera port from the level of the umbilicus and two $5 \mathrm{~mm}$ trocars from the right and left lumbar regions. Initial exploration showed that the patient had a Morgagni hernia situated on the right and omentum and colon were herniated through the defect to the thoracic cavity. The defect was $5 \times 6 \mathrm{~cm}$ in diameter. The contest were reduced in to the abdomen and the defect was continuously closed by $2 / 0$ polypropylene suture. The repair was reinforced with $6 \times 6$ $\mathrm{cm}$ dual mesh using endoscopic mesh fixators (Tacker). The patient was discharged on the postoperative $3^{\text {rd }}$ day without any problems. The postoperative $6^{\text {th }}$ month control was uneventful.

\section{Discussion}

Symptomatology of the Morgagni hernia is governed by the size of the defect and the contents of the hernia sac. ${ }^{[4]}$
Abdominal pain and constipation are common symptoms. Furthermore, right upper quadrant blunt abdominal pain may also be observed in the patients. Complete obstruction and strangulation of the herniating bowel segments are extremely rare. Loong et al. ${ }^{[5]}$ have reported a series of 140 patients and 19 patients presented with signs of acute intestinal obstruction. In the present case there has been signs and symptoms of partial intestinal obstruction for the last 3 years before admission to the emergency department. Therefore in patients with dyspeptic complaints and constipation the diagnosis of Morgagni hernia should always be kept in mind and necessary diagnostic precautions should be taken.

Plain chest roentgenograms show para-cardiac mediastinal mass and air filled intestinal segments in the thorax. However, in cases with intermittent complaints the radiologic evaluation can completely be normal.

Thorax computerized tomography, abdominal ultrasound and barium enhanced plain abdominal films as well as diagnostic thoracoscopy and laparoscopy may be other diagnostic modalities ${ }^{[6]}$ In the present case report plain chest roentgenogram showed mediastinal mass with air densities inside and plain abdominal films showed signs of intestinal obstruction. The thorax computerized tomography showed colon and omentum in the hernia sac.

Surgical therapy should be considered in all pediatric patients and in all symptomatic adult patients. Surgical therapy in asymptomatic adults is a controversial point. ${ }^{[4]}$ However, studies on the subject have stated that surgical therapy should be employed in asymptomatic cases as well because there is a risk of incarceration and strangulation. ${ }^{[4,5]}$ Especially in cases where there are bowel
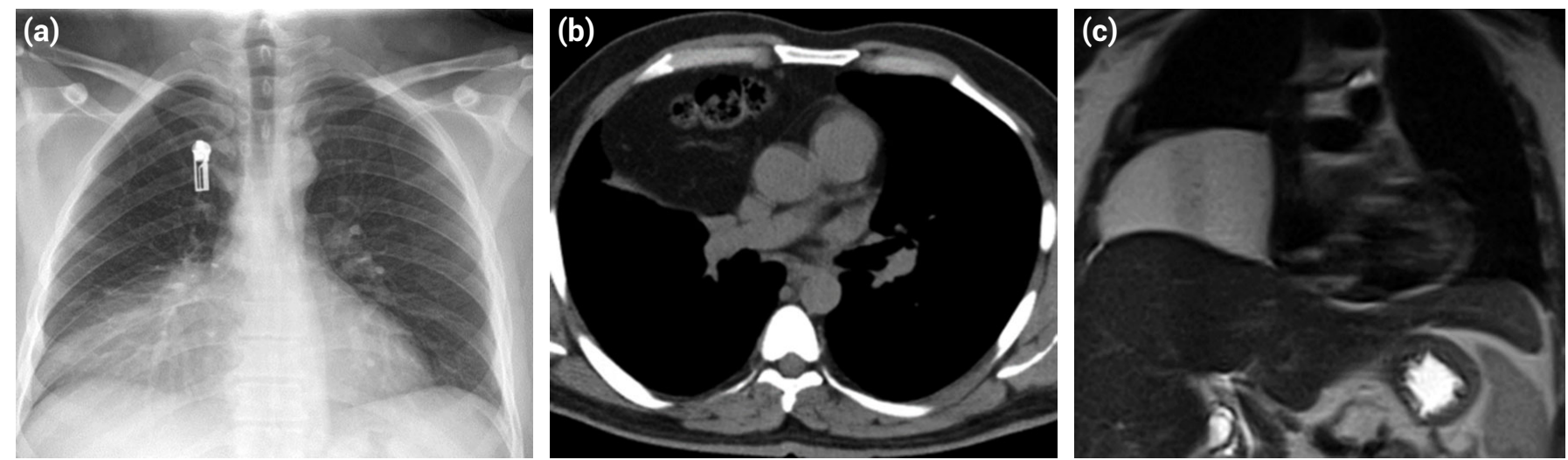

Figure 1. (a) Postero-anterior plain chest roentgenogram showing density in the right lower thoracic cavity with air densities inside (red circle). (b) Thorax computerized tomography axial sections showing paracardiac hernia showing colon and adipose tissue densities inside (red arrow). (c) Coronal reconstruction showing hernia sac extending towards the level of the carina (red arrow head). 
segments in the hernia sac, there is increased risk of intestinal obstruction If the hernia sac is small and contains omental structures then the patient can be followed up conservatively. Nevertheless, in patients with persistent symptoms and reduced quality of life surgical therapy should be considered. ${ }^{[7]}$

In the present case the intestinal obstruction was due to herniation of the colon and omental structures. laparoscopic surgery or open surgery can be performed but laparascopic surgery is more advantageous in terms of blood loss and length of hospital stay. So we opted for laparoscopic surgery, no complications developed and discharged the patient 2 days later.

In $90 \%$ of the cases a hernia sac is present. There is no consensus whether the hernia sac should resected. Loong et al. ${ }^{[5]}$ have reported that they have not excised the hernia sac and have not encountered any problems in the postoperative period. Kuster et al. ${ }^{[6]}$ have suggested not to resect hernia sac because it may lead to pneumomediastinum that ay result in fatal circulatory complications. Rau et al. ${ }^{[8]}$ have suggested to resect the hernia sac in order to avoid development of postoperative cyst and lobulated mediastinal collection. Ramanchandran et al. ${ }^{[9]}$ have reported that they have not resected hernia sac in their case series and have reported no postoperative problem related to retained hernia sac and they reported that there were no sac and related structures in the postoperative 1st month. In the present case we did not resect the hernia sac because it was adherent to pericardium and vital mediastinal structures.

In conclusion, Morgagni hernia should be suspected in any case with dyspepsia and constipation and proper investigative measures should be taken. Surgical therapy should be considered in any patient with Morgagni hernia because of the risk of intestinal obstruction and other complications. Resection of the hernia sac may not be possible, especially in cases whom the hernia sac is adherent to the vital structures in the mediastinum. Primary repair with mesh reinforcement seems to be reasonable surgical strategy. Laparoscopic morgagni hernia repair is a technically feasible procedure for surgeons experienced.

\section{Disclosures}

Informed Consent: Written informed consent was obtained from the patient for the publication of the case report and the accompanying images.

Peer-review: Externally peer-reviewed.

Conflict of Interest: None declared.

\section{References}

1. Losanoff JE, Sauter ER. Congenital posterolateral diaphragmatic hernia in an adult. Hernia 2004;8:83-5. [CrossRef]

2. Goh BK, Teo MC, Chng SP, Soo KC. Right-sided Bochdalek's hernia in an adult. Am J Surg 2007;194:390-1. [CrossRef]

3. Comer TP, Clagett OT. Surgical treatment of hernia of the foramen of Morgagni. J Thorac Cardiovasc Surg 1966;52:461-8.

4. Barut I, Tahran OR, Cerci C, Akdeniz Y, Bulbul M. Intestinal obstruction caused by a strangulated Morgagni hernia in an adult patient. J Thorac Imaging 2005;20:220-2. [CrossRef]

5. Loong TP, Kocher HM. Clinical presentation and operative repair of hernia of Morgagni. Postgrad Med J 2005;81:41-4.

6. Kuster GG, Kline LE, Garzo G. Diaphragmatic hernia through the foramen of Morgagni: laparoscopic repair case report. J Laparoendosc Surg 1992;2:93-100. [CrossRef]

7. Yörük Y, Mamedov R, Köse S, Ekim T. Eripkin Morgagni hernilerinde cerrahi tedavi. GKDC Dergisi 1998;6:517-20.

8. Rau HG, Schardey HM, Lange V. Laparoscopic repair of a Morgagni hernia. Surg Endosc 1994;8:1439-42. [CrossRef]

9. Ramachandran CS, Vijay A. Laparoscopic transabdominal repair of hernia of Morgagni-Larrey: brief clinical reports. Surg Laparosc Endosc 1999;9:358-65. [CrossRef] 